\title{
Comparative Investigation of Single Voxel Magnetic Resonance Spectroscopy and Dynamic Contrast Enhancement MR Imaging in Differentiation of Benign and Malignant Breast Lesions in a Sample of Iranian Women
}

\author{
Fariborz Faeghi $^{1}$, Banafsheh Baniasadipour ${ }^{1 *}$, Jalal Jalalshokouhi ${ }^{2}$
}

\begin{abstract}
Purpose: To make a comparison of single voxel magnetic resonance spectroscopy (SV-MRS) and dynamic contrast enhancement (DCE) MRI for differentiation of benign and malignant breast lesions in a sample of Iranian women. Materials and Methods: A total of 30 women with abnormal breast lesions detected in mammography, ultrasound, or clinical breast exam were examined with DCE and SV-MRS. tCho (total choline) resonance in MRS spectra was qualitatively evaluated and detection of a visible tCho peak at $3.2 \mathrm{ppm}$ was defined as a positive finding for malignancy. Different types of DCE curves were persistent (type 1), plateau (type 2), and washout (type 3). At first, lesions were classified according to choline findings and types of DCE curve, finally being compared to pathological results as the standard reference. Results: this study included 19 patients with malignant lesions and 11 patients with benign ones. While $63.6 \%$ of benign lesions ( 7 of 11) showed type 1 DCE curves and 36.4\% (4 of 11) showed type 2, 57.9\% (110f 19) of malignant lesions were type 3 and $42.1 \%$ (8 of 19) type 2. Choline peaks were detected in 18 of 19 malignant lesions and in 3 of 11 benign counterparts. 1 malignant and 8 benign cases did not show any visible resonance at $3.2 \mathrm{ppm}$ so SV-MRS featured $94.7 \%$ sensitivity, $72.7 \%$ specificity and $86.7 \%$ accuracy.Conclusions: The present findings indicate that a combined approach using MRS and DCE MRI can improve the specificity of MRI for differentiation of benign and malignant breast lesions.
\end{abstract}

Keywords: Magnetic resonance spectroscopy - dynamic contrast enhancement MRI - breast lesions - benign -malignant

Asian Pac J Cancer Prev, 16 (18), 8335-8338

\section{Introduction}

Breast cancer is one of the leading causes of death in women all over the world and its prevalence is increasing in many countries. It's the most common cancer in Iranian women with a mortality rate of 2614 cases per year (Jafari et al., 2014; Sharifian et al., 2015). Early detection of that would be helpful for its successful treatment.

Nowadays some techniques such as X-ray mammography, ultrasound and magnetic resonance imaging (MRI) are used for the diagnosis of breast lesions (Kvistad et al., 1999; Katz-Brull et al., 2002). Mammography which is the most common screening method of this disease, has high sensitivity but low specificity especially in low-density breasts. False positive results of mammography is about $60-80 \%$, resulting in many unnecessary biopsies of benign lesions and in some patients there is the likelihood of infections, bleeding and abscess because of biopsy, also the risk of complications from general anesthesia in patients for whom local anesthesia is not possible (Huang et al., 2004).

Specificity of ultrasonography to classify benign and malignant lesions has been reported about 30\% (Katz-
Brull et al., 2002).

In recent years, the results of various studies have shown that the use of MRI techniques has improved the detection and assessment of breast lesions (Huang et al., 2004).

Compared to other imaging techniques, MRI because of its high soft tissue contrast, the use of thin slices and multi-planar scanning feature, can display different lesions better (Orel, 1999).

Dynamic Contrast Enhanced (DCE) MRI is a method which increases the specificity of MR imaging for diagnosis of breast cancer. It has Specific patterns of enhancement, persistent (type 1), plateau (type 2), and washout (type 3). Type 1 has a steady increase, type 2 reaches to a constant level, and type 3 has an initial peak and after that it has an immediate decrease in the signal intensity. Usually benign lesions show "persistent" pattern and malignant lesions show "wash out" pattern but "plateau" can be seen in both kind of lesions. Although malignant lesions have an early wash out and more rapid increase in signal intensity but there are some exceptional cases which lead to overlapping in diagnosis of benign and malignant lesions so additional diagnostic methods 
are needed (Huang et al., 2004; Jacobs et al., 2005).

Proton magnetic resonance spectroscopy (1H-MRS) is a valuable diagnostic technique presenting metabolic information of breast lesions (Tan et al., 2015).

Breast cancer studies by MRS show that choline level in malignant lesions is higher than that in benign ones or normal breast tissue (Kousi et al., 2012). choline peak ,centered at 3.2 parts per million, includes choline, phosphocholine, and glycerophosphocholine, referred together as total choline (tCho) which is a marker of increased membrane synthesis so it can be a sign of cancer (Baltzer and Dietzel, 2013; Melsaether and Gudi, 2014). when normal condition of cells turns into a malignant state, tCho peak would be affected by metabolic changes of cell membrane and it's mainly because of phosphocholine variations (Begley et al., 2012).

Thus, adding this technique to breast MR examination can be helpful for differentiation of those lesions that their enhancement patterns are equivalent.

\section{Materials and Methods}

Patients. 35 women were referred for MR evaluation of abnormal breast lesions detected in mammography, ultrasound, or clinical breast exam. None of them were in lactation or pregnancy period because choline can be detected in lactating breasts, too. Furthermore, during pregnancy and lactating, Breast parenchyma is exposed to the hormonal changes and shows abnormal uptake of contrast material (Fischer, 2004b; Kousi et al., 2012).

For better evaluation of DCE curve, MRI exam was acquired in the second week of the menstrual cycle. in the first and fourth week, Estrogen hormone changes lead to Increased vascular permeability so abnormal diffused or focal enhancement would happen (Fischer, 2004a; Hendrick, 2008a; Hendrick, 2008b).

5 patients with lesions smaller than $2 \mathrm{~cm}$ in diameter (smaller than the voxel size that we used in MRS exam) were excluded from this study.

After MR examinations, biopsies were performed on all patients and according to the pathological result, malignant and benign lesions were classified.

Informed consent was received from all contributors in this study.

MRI acquisition. MRI and 1H-MRS exams were acquired in the prone position for minimizing respiratory motion of the breasts, on a 1.5 Tesla MRI System (Avanto; Siemens Medical Solutions, Erlangen, Germany). A dedicated phased array breast coil was used for both MRI and MRS sequences.

MRI protocol included 3plane localizer, axial T2 FSE (Fast Spin Echo, TR/TE $=3200 / 110 \mathrm{msec}$, slice thickness $=3.5 \mathrm{~mm}, \mathrm{ETL}=12$, matrix $=256 * 284$, $\mathrm{BW}=205 \mathrm{~Hz}, \mathrm{FOV}=350 \mathrm{~mm}, \mathrm{NSA}=2$ ), T2 TIRM (Turbo Inversion Recovery Magnitude, TR/TE $=4500 / 70 \mathrm{msec}$, $\mathrm{TI}=160$, slice thickness $=4 \mathrm{~mm}, \mathrm{ETL}=16$, atrix $=256 * 284$, $\mathrm{BW}=192 \mathrm{~Hz}, \mathrm{FOV}=350 \mathrm{~mm}, \mathrm{NSA}=4)$ and T2.FSE.FS (TR/ $\mathrm{TE}=4100 / 100 \mathrm{msec}$, slice thickness $=3.5 \mathrm{~mm}, \mathrm{ETL}=13$, matrix $=320 * 384, \mathrm{BW}=192 \mathrm{~Hz}, \mathrm{FOV}=350 \mathrm{~mm}, \mathrm{NSA}=2$ ).

T1 DCE images were acquired using T1-3D FLASH (Fast Low Angle Shot, TR/TE= 4.3/1.3msec, slice thickness $=1 \mathrm{~mm}$, matrix $=323 * 448, \mathrm{BW}=350 \mathrm{~Hz}$, $\mathrm{FOV}=350 \mathrm{~mm}, \mathrm{NSA}=1, \mathrm{FA}=20$ ). Gadolinium was automatically injected with an injection rate of $3 \mathrm{ml} / \mathrm{s}$ and concentration of $0.1 \mathrm{mmol} / \mathrm{kg}$. The temporal resolution was 30 seconds. Then the different series of dynamic images were transferred to mean curve software, the second series were selected and ROI was manually depicted on the high signal region of tumors. DCE curves were drawn by software.

MRS examination. SV-MRS (single voxel MRS) was performed using a point-resolved spectroscopy sequence (PRESS), parameters were TR/TE $=1620 / 270$, voxel size $=15 * 15 * 15 \mathrm{~mm} 3$, acquisitions $=256$, spectral width $=1000 \mathrm{~Hz}$, data points $=1024$, and the time of acquisition was $7 \mathrm{~min}$. a long TE was used to increase the detection of tCho resonance because of the longer T2 of tCho ( $>350 \mathrm{msec}$ ) compared with T2 of fat (about $100 \mathrm{msec}$ ) (Sardanelli et al., 2009; Kousi et al., 2012). Automatic Shimming was performed followed by manual shimming on the water resonance for optimization of the homogeneity on all of VOIs (voxels of interest). Water peak line-widths of 10 to $20 \mathrm{~Hz}$ (full width at halfmaximum; FWHM) were achieved. After shimming, spectra were acquired with water suppression by applying CHESS (three chemical shift selective) excitation pulses and the bandwidth of each pulse was $60 \mathrm{~Hz}$. MRS examinations were acquired after contrast administration and subtraction images were used to determine the voxel position .Voxel size should be adapted to the tumor size. Large voxel or poor positioning of that, lead to including adipose and fibro-glandular tissue, so tCho peak would be reduced because of partial volume effect.

\section{Statiscal and data analysis:}

Cho resonance in MRS spectra was qualitatively evaluated and the detection of a visible tcho peak at $3.2 \mathrm{ppm}$ was defined as a positive finding for malignancy (Cecil et al., 2001; Jacobs et al., 2005).

For dynamic MR images, lesions that showed a washout or plateau curve Were assumed malignant and Lesions with delayed or indeterminate enhancement, showing persistent curve, were assumed benign (Kuhl et al., 1999; Jacobs et al., 2005).

Fisher's exact test was used to evaluate the significance of the curve type distribution in benign and malignant lesions. (Kuhl et al., 1999)

\section{Results}

Pathological evaluation showed that 19 patients had malignant lesions and 11 patients had benign ones. These results were used as reference standard. $63.6 \%$ of benign lesions ( 7 of 11 ) showed type 1 of DCE curve and $36.4 \%$ (4of 11) showed type $2.57 .9 \%$ (11 of 19) of malignant lesions showed type 3 and $42.1 \%$ (8 of 19) showed type 2 . if type 2 and 3 be assumed as malignant cases, therefore sensitivity, specifity and accuracy of DCE MRI according to the curve type would be $100 \%, 63.6 \%$ and $86.7 \%$ respectively.

Choline peak was detected in 18 of 19 malignant lesions and in 3 of 11 benign lesions. One malignant and 
Single Voxel MRS and Dynamic Contrast Enhancement MR in Differentiating Benign from Malignant Breast Lesions in Iran

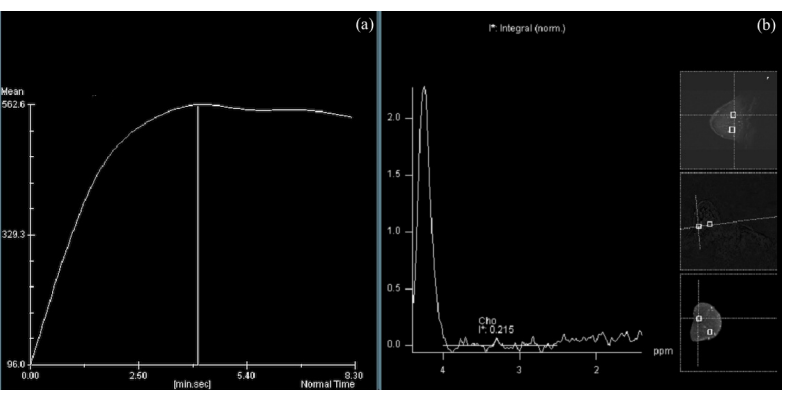

Figure 1. A 33-year-old patient with a benign lesion. (a) DCE curve shows a plateau pattern (b) high choline peak is not detected in $\mathrm{mr}$ spectra of this lesion

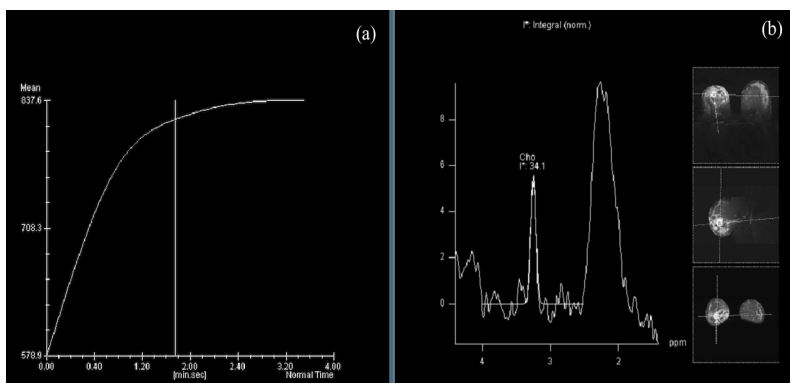

Figure 2. A 52-year-old Patient with a Malignant

Lesion. (a) DCE curve shows a plateau pattern. (b) high choline peak is detected in $\mathrm{mr}$ spectra of this lesion

Table 1. Choline Finding, DCE Curve type and Pathological Results

\begin{tabular}{|c|c|c|c|}
\hline & $\begin{array}{l}\text { choine } \\
\text { finding }\end{array}$ & $\begin{array}{c}\text { Type of } \\
\text { DCE curve }\end{array}$ & $\begin{array}{c}\text { pathologic } \\
\text { result }\end{array}$ \\
\hline 1 & + & 3 & + \\
\hline 2 & - & 2 & - \\
\hline 3 & + & 3 & + \\
\hline 4 & + & 3 & + \\
\hline 5 & + & 3 & + \\
\hline 6 & + & 2 & + \\
\hline 7 & - & 1 & - \\
\hline 8 & + & 3 & + \\
\hline 9 & + & 2 & + \\
\hline 10 & - & 1 & - \\
\hline 11 & - & 1 & - \\
\hline 12 & + & 3 & + \\
\hline 13 & - & 1 & - \\
\hline 14 & + & 2 & + \\
\hline 15 & + & 3 & + \\
\hline 16 & + & 2 & + \\
\hline 17 & + & 2 & + \\
\hline 18 & + & 2 & + \\
\hline 19 & + & 2 & + \\
\hline 20 & + & 3 & + \\
\hline 21 & + & 3 & + \\
\hline 22 & + & 2 & - \\
\hline 23 & + & 2 & - \\
\hline 24 & + & 1 & - \\
\hline 25 & + & 3 & + \\
\hline 26 & + & 2 & + \\
\hline 27 & - & 2 & - \\
\hline 28 & - & 3 & + \\
\hline 29 & - & 1 & - \\
\hline 30 & - & 1 & - \\
\hline
\end{tabular}

8 benign cases did n't show any visible resonance at 3.2 ppm so SV-MRS represents $94.7 \%$ sensitivity ,72.7\% specificity and $86.7 \%$ accuracy.

Table 1 shows choline findings, curve types and pathologic results of 30 patients.

Figure 1 shows a benign lesion with plateau curve and negative choline finding and Figure 2 shows a malignant lesion with plateau curve and positive choline finding.

Based on the Fisher's Exact Test, there is a significant difference between the distribution of the curve types in benign and malignant lesions $(\mathrm{P}<0.001)$.

\section{Discussion}

Breast cancer is one of the most common malignancies among Iranian women (Mousavi et al., 2007). Early detection and characterization of breast lesions, is very effective for reducing the mortality rate of this cancer. In recent years, non-invasive techniques for evaluation of breast cancer have become more prevalent. (Stanwell et al., 2005)

Spectroscopy is a non-invasive method, representing chemical information from a selected region in the body. In the in vivo 1H MR spectroscopy of the breast, choline centered at $3.2 \mathrm{ppm}$, is the most important metabolite. It's detectable in breast cancers and generally undetectable in normal breast tissue. the increased level of tCho in breast cancer is related to increased membrane synthesis. (Baek et al., 2008; Menezes et al., 2014)

In the present study, tCho was found in 18 of 19 malignant lesions but it was detected in 3 of benign ones, too. The elevated tCho peak is associated with increased cellular turnover in breast carcinoma but benign lesions such as proliferative fibroadenomas may also represent choline signal (Baek et al., 2008). Therefore, the specificity of MRS can be decreased because of these benign cases.

In previous MRS studies, Kvistad et al and Yeung et al found tCho in some cases of fibroadenoma (Kvistad et al., 1999; Yeung et al., 2001). Also, Mackinnon et al had a research on MRS of fine-needle breast biopsy specimens and reported detectable levels of tCho in 3 of the 15 fibroadenomas. (Mackinnon et al., 1997) tCho can be detected in fibrocystic disease and tubular adenoma, too. (Roebuck et al., 1998; Cecil et al., 2001)

We had one malignant case which didn't show tCho peak. This can be because of poor voxel positioning or mismatch between voxel and lesion size, so normal and adipose tissue could be included, too. Lipid contamination disrupts the uniformity of the field inside the voxel and makes the resonance peaks broad. Wings or sidebands of the residual water and lipid signals limit the detection of small choline resonance. (Bolan et al., 2002; Barker et al., 2009)The spectral quality may also be decreased by patient's respiratory motion (Bolan et al., 2004)

$36.4 \%$ of benign lesions and $42.1 \%$ of malignant lesions represent type 2 of DCE curve, so differentiation of lesions with this type of curve is not possible.

Of 12 lesions with type 2, Choline peak was detected in 8 malignant ones and was not detected in 2 benign ones. So SV-MRS can improve specificity of DCE-MRI 
for characterization of breast lesions.

We had some limitations such as small populations, qualitative evaluation of choline peak, partial volume effect and limited spatial coverage. For further studies, we suggest using multi-voxel MRS to evaluate large heterogeneous or multiple lesions. Using high tesla magnets, more specialized coils and quantitative analysis of choline resonance can improve spectroscopy results of breast.

Conclusion: The present findings indicate that a combined approach using MRS and DCE MRI can improve the specificity of MRI for differentiation of benign and malignant breast lesions.

\section{References}

Baek HM, Chen JH, Yu HJ, et al (2008). Detection of choline signal in human breast lesions with chemical-shift imaging. J Magnetic Resonance Imaging, 27, 1114-21.

Baltzer PA, Dietzel M (2013). Breast lesions: diagnosis by using proton MR spectroscopy at 1.5 and 3.0 T-systematic review and meta-analysis. Radiol, 267, 735-46.

Barker PB, Bizzi A, De Stefano N, et al (2009). MRS in breast cancer. In 'Clinical MR spectroscopy: techniques and applications', Eds Cambridge University Press, 229-42

Begley JK, Redpath TW, Bolan PJ, et al (2012). In vivo proton magnetic resonance spectroscopy of breast cancer: a review of the literature. Breast Cancer Res, 14, 207.

Bolan PJ, DelaBarre L, Baker EH, et al (2002). Eliminating spurious lipid sidebands in $1 \mathrm{H}$ MRS of breast lesions. Magn Reson Med, 48, 215-22.

Bolan PJ, Henry PG, Baker EH, et al (2004). Measurement and correction of respiration-induced B0 variations in breast $1 \mathrm{H}$ MRS at 4 Tesla. Magn Reson Med, 52, 1239-45.

Cecil KM, Schnall MD, Siegelman ES, et al (2001). The evaluation of human breast lesions with magnetic resonance imaging and proton magnetic resonance spectroscopy. Breast Cancer Res Treatm, 68, 45-54.

Fischer U (2004a). normal findings in MR mammography. In 'Practical MR mammography', Eds Thieme, 41-50

Fischer U (2004b). technique and methods In 'Practical MR mammography', Eds Thieme, 11-21

Hendrick RE (2008a). breast magnetic resonance imaging aquisition protocols. In 'Breast MRI: Fundamentals and Technical Aspects', Eds 69-135

Hendrick RE (2008b). contrast agents in breast magnetic resonance imaging In 'Breast MRI: Fundamentals and Technical Aspects', Eds 113-34

Huang W, Fisher PR, Dulaimy K, et al (2004). Detection of Breast Malignancy: Diagnostic MR Protocol for Improved Specificity 1. Radiol, 232, 585-91.

Jacobs MA, Barker PB, Argani P, et al (2005). Combined dynamic contrast enhanced breast MR and proton spectroscopic imaging: a feasibility study. J Magn Reson Imag, 21, 23-8.

Jafari M, Nakhaee N, Goudarzi R, et al (2014). Participation of the Women Covered by Family Physicians in Breast Cancer Screening Program in Kerman, Iran. Asian Pac J cancer prevention, 16, 4555-61.

Katz-Brull R, Lavin PT, Lenkinski RE (2002). Clinical utility of proton magnetic resonance spectroscopy in characterizing breast lesions. J National Cancer Inst, 94, 1197-203.

Kousi E, Tsougos I, Vasiou K, et al (2012). Magnetic resonance spectroscopy of the breast at 3T: pre-and post-contrast evaluation for breast lesion characterization. Scientific world $J, 2012$.
Kuhl CK, Mielcareck P, Klaschik S, et al (1999). Dynamic breast mr imaging: Are signal intensity time course data useful for differential diagnosis of enhancing lesions? 1. Radiol, 211, 101-10.

Kvistad KA, Bakken IJ, Gribbestad IS, et al (1999). Characterization of neoplastic and normal human breast tissues with in vivo $1 \mathrm{H}$ MR spectroscopy. J Magn Reson Imaging, 10, 159-64.

Mackinnon WB, Barry PA, Malycha PL, et al (1997). Fine-needle biopsy specimens of benign breast lesions distinguished from invasive cancer ex vivo with proton MR spectroscopy. Radiol, 204, 661-6.

Melsaether A, Gudi A (2014). Breast Magnetic resonance imaging performance: safety, techniques, and updates on diffusion-weighted imaging and magnetic resonance spectroscopy. Topics Magn Reson Imaging, 23, 373-84.

Menezes GL, Knuttel FM, Stehouwer BL, et al (2014). Magnetic resonance imaging in breast cancer: A literature review and future perspectives. World J Clinical Oncol, 5, 61.

Mousavi SM, Montazeri A, Mohagheghi MA, et al (2007). Breast cancer in Iran: an epidemiological review. Breast $J, 13,383-91$.

Orel SG (1999). Differentiating Benign from Malignant Enhancing Lesions Identified at MR Imaging of the Breast: Are Time-Signal Intensity Curves an Accurate Predictor? 1. Radiol, 211, 5-7.

Roebuck JR, Cecil KM, Schnall MD, et al (1998). Human breast lesions: characterization with proton MR spectroscopy. Radiol, 209, 269-75.

Sardanelli F, Fausto A, Di Leo G, et al (2009). In vivo proton MR spectroscopy of the breast using the total choline peak integral as a marker of malignancy. AmJ Roentgenol, 192, 1608-17.

Sharifian A, Pourhoseingholi MA, Emadedin M, et al (2015). Burden of Breast Cancer in Iranian Women is Increasing. Asian Pac J Cancer Prev, 16, 5049-52.

Stanwell P, Gluch L, Clark D, et al (2005). Specificity of choline metabolites for in vivo diagnosis of breast cancer using $1 \mathrm{H}$ MRS at 1.5 T. Eur Radiol, 15, 1037-43.

Tan J, Xu L, Yao W, et al (2015). In vivo post-contrast 1H-MRS evaluation of malignant and benign breast lesions: a metaanalysis. Tumor Biol, 36, 345-52.

Yeung DKW, Cheung HS, Tse GMK (2001). Human Breast Lesions: Characterization with Contrast-enhanced in Vivo Proton MR Spectroscopyac"Initial Results 1. Radiol, 220, 40-6. 